Draft Version June 9, 2014

Preprint typeset using $\mathrm{AT}_{\mathrm{E} X} \mathrm{X}$ style emulateapj v. 11/10/09

\title{
A SIMPLIFIED APPROACH TO UNCERTAINTY QUANTIFICATION FOR ORBITS IN IMPULSIVE DEFLECTION SCENARIOS
}

\author{
Kirsten Howley \& Joseph WAsem ${ }^{1}$ \\ Draft version June 9, 2014
}

\begin{abstract}
For the majority of near-Earth Asteroid (NEA) impact scenarios, optimal deflection strategies use a massive impactor or a nuclear explosive, either of which produce an impulsive change to the orbit of the object. However, uncertainties regarding the object composition and the efficiency of the deflection event lead to a non-negligible uncertainty in the deflection delta-velocity. Propagating this uncertainty through the resulting orbit will create a positional uncertainty envelope at the original impact epoch. We calculate a simplified analytic evolution for impulsively deflected NEAs and perform a full propagation of uncertainties that is nonlinear in the deflection delta-velocity vector. This provides an understanding of both the optimal deflection velocities needed for a given scenario, as well as the resulting positional uncertainty and corresponding residual impact probability. Confidence of a successful deflection attempt as a function of launch opportunities is also discussed for a specific case.
\end{abstract}

\footnotetext{
Subject headings: Asteroids: dynamics, individual (2011 AG5); Comets: dynamics; Orbit: determination, uncertainty; Satellites: dynamics; Mission planning
}

\section{INTRODUCTION}

Throughout its history, Earth has been impacted by passing asteroids and comets (Alvarez et al. 1980; Hildebrand et al. 1991). Collisions have played an important role in Earth's evolution and are cited as the main delivery mechanism for water (Owen \& Bar-Nun 1995; Morbidelli et al. 2000), the progenitor for moon (Ćuk \& Stewart 2012, and references therein) and the source of several mass extinctions (Alvarez \& Muller 1984, and references therein). The present day population of asteroids passing near Earth's orbit are called near-Earth Asteroids (NEAs). It is hypothesized most that near-Earth Asteroids (NEAs) are main-belt objects that were perturbed into Earth-crossing orbits through both gravitational and non-gravitational effects (Binzel et al. 1992; Bottke et al. 2002).

Over the last hundred years there have been numerous small, but notable, NEA events: the 2013 Chelyabinsk meteor ( $20 \mathrm{~m}$, JPL 2013), the 1963 Price Edward Island event (Edwards et al. 2006), and the 1908 Tunguska event ( $50 \mathrm{~m}$, Shoemaker 1983, Boslough \& Crawford 2008). The probability of a strike by another small NEA over the course of the next century is relatively high, while large asteroid and comet collisions with Earth represent a much lower-probability but potentially higherconsequence threat (NRC 2010). Asteroids with absolute magnitudes $(H) 22.0$ or brighter $(\gtrsim 150$ meter diameter) and minimum orbit intersection distances (MOIDs) less than $0.05 \mathrm{AU}(\sim 20$ lunar distances $)$ are classified as Potentially Hazardous Asteroids (PHAs). According to the International Astronomical Union Minor Planet Center, at least 600 PHAs will pass near the Earth over the next century (IAU 2014). An illustration of the PHA

\footnotetext{
${ }^{1}$ Lawrence Livermore National Laboratory, P.O. Box 808, Livermore, CA 94551; [howley1, wasem2] @llnl.gov; +1(925) 4221100
}

population and its orbital characteristics over the next century is shown in Fig. 1, with special attention given to objects with known diameters over $270 \mathrm{~m}$ in size (i.e. Apophis-size and greater). Note, the small population of near-Earth comets (NECs) are not included in Fig. 1. While this is another important class of objects, they are extremely few in number compared to asteroids and have less predictable orbits due to velocity changes from outgassing and gravitational perturbations (Yeomans et al. 2004).

Over the last few decades, there has been increased interest in developing mitigation strategies to deal with the potential threat (Morrison 1992; NASA 2005, 2008; Holdren 2010; NRC 2010). For scenarios where the object is large or there is little lead time, kinetic impactors and/or nuclear explosives are the most efficient means of providing the necessary change in velocity to an NEA to avoid an impact (Ahrens \& Harris 1992; Shafer et al. 1994; NASA 2007). Both of these deflection methods provide a sudden impulse to the NEA, where the orbital parameters governing the motion of the object are changed on timescales that are much shorter than orbital evolution timescales. As such, these changes can be approximated to an extremely high degree as being instantaneous, taking an object with an initial set of orbital parameters and moving it to a new set. If the intended outcome is to deflect the object (as opposed to disruption), then velocity changes less than the escape velocity (typically of order a few $\mathrm{cm} \mathrm{s}^{-1}$ ) are desirable, since above this limit the likelihood of breaking the object up is dramatically increased for most object compositions.

A variety of uncertainty sources are present in any mitigation scenario. The orbital parameters of the object will be known only with limited accuracy, and uncertainties in the deflection delta-velocity resulting from uncertainties in the magnitude and direction of the impulse, energy coupling efficiency, and the properties of the target 


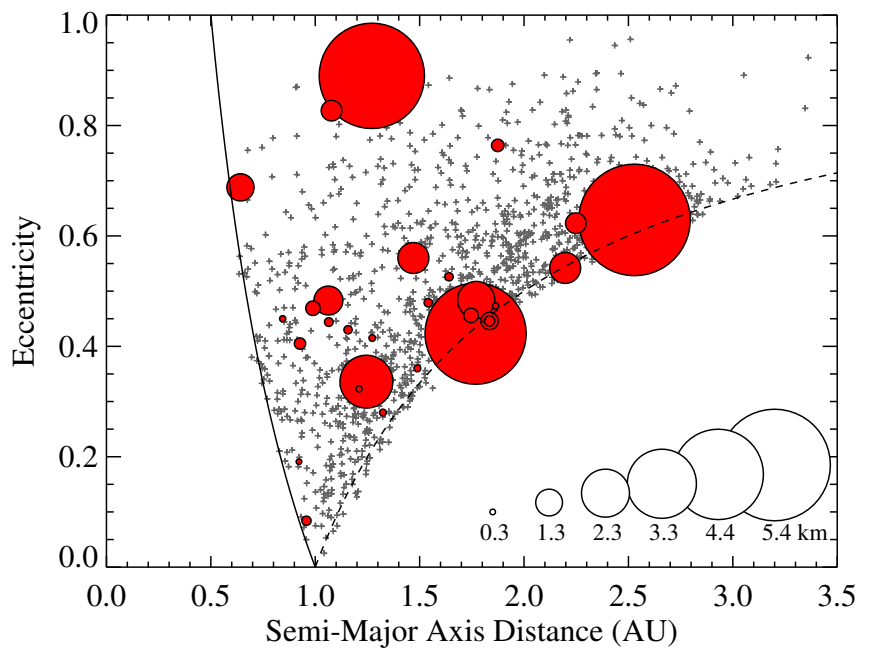

Figure 1. Semi-major axes and eccentricities of known PHAs. Red circles show a subset of PHAs with known diameters greater than 270 meters (the size scale is shown on the lower right) that will have a close-approach with Earth over the next century. The solid line on the left denotes orbits that reach $1 \mathrm{AU}$ at aphelion, the dashed line on the right denotes orbits that reach $1 \mathrm{AU}$ at perihelion. The largest three circles, from right to left: Phaethon, Florence and Toutatis. Data courtesy of IAU (2014) and JPL (Small-Body Database Search Engine).

(mass, composition, porosity, etc.) introduce additional uncertainties. For example, in the case of an impact or nuclear explosion, slight changes in the angle of approach of the spacecraft and/or detonation distance (for the nuclear case) will affect the magnitude and direction of the resulting deflection. Furthermore, the magnitude of the deflection will be influenced by the properties of the target. A porous target has a lower energy transfer efficiency than a non-porous target (Holsapple \& Housen 2012). Errors in the total mass estimate of the NEA also lead to uncertainties in the final deflection delta-velocity, since the deflection delta-velocity goes as $p / m$, where $p$ is the momentum imparted to the NEA and $m$ is the mass of the NEA minus the material ejected (with equal and opposite momentum $-p$ ) as a result of the impulse. These examples illustrate a subset of the sources of error due to uncertainties in the properties of the object and/or the accuracy of the mission planning that must be considered when planning a deflection mission. In a deflection attempt these orbital uncertainties propagate through to the new, perturbed orbit and contribute to an uncertainty in the final position at the original impact epoch.

In this paper, we extend the work of Burns (1976) and present a simplified, non-linear analytic determination of the effect an instantaneous, planar change in velocity has on an orbit, and examine the propagation of uncertainties in this velocity change through the resulting orbit. We approximate the motion using the two body problem where keyholes, interactions with planets, gravitational influence by Earth in the final approach, etc. are not considered. We believe that consideration of these important, higher order effects are best studied using detailed N-body calculations.

The purpose of this work is to provide a quick scoping tool that translates uncertainties in velocity changes due to a deflection attempt into uncertainties in position at predefined times in the orbit. While there are several already existent methods for calculating orbits and changes to those orbits, most are difficult for a nonspecialist to use and/or are computationally expensive. Direct numerical simulations of N-body interactions, as stated above, provide the most complete solution to the problem but are numerically tricky to get right and usually require more sophisticated numerical techniques beyond the standard Runge-Kutta integrators. Applying Monte Carlo techniques to a stable numerical integrator provides the necessary uncertainty quantification, but this can get prohibitively expensive and again relies on the underlying stability of the numerical integrator. One can always simplify this approach to the 2-body case, but then one is usually left with the determination that the analytical approach, defined here, is superior. Again, we stress that the fully numerical N-body approach is best for detailed studies, but that it is overkill for most quick scoping studies, which is what the tool derived in this paper aims to address.

There are also analytical methods available that do not require the same level of computational power. The most widely used is the simple linear approximation created by Aherns et al. However, while this approach obtains results that are correct in an orbit-average sense, it does not account for variations within each orbit. As shown below, these can be factors of $2 \mathrm{x}$ in the required deflection delta-velocity and when coupled with mission planning scenarios this difference can become appreciable. A more recent analytical approach (Johnson 2010; Sherrill et al. 2014, and refs therein) using elliptical HillClohessy-Wiltshire equations is a promising alternative approach. However, in this paper we are seeking an approach that non-experts in astrodynamics could easily understand and utilize, in an effort to reach the broad and very diverse backgrounds of the asteroid deflection community.

This type of analysis is useful in a number of applications, for example, in exploring how deflection deltavelocity uncertainties propagate over large orbit parameter spaces. This analysis can also be used to inform mesh resolution in hydrodynamic simulations that model how asteroidal materials respond to nuclear and impact deflection attempts. Since coarser mesh resolution translates into larger uncertainties in simulated deflection velocities, an upper bound on the mesh resolution can be estimated by setting an allowable uncertainty in the final position of the deflected object and translating that into an allowable uncertainty in the deflection delta-velocity. In fact, this latter point was the initial motivation for this work and will be addressed in more detail in an upcoming paper.

This paper is outlined as follows. We begin in $\S 2$ by summarizing the restricted two-body equations, and use these to derive the non-linear change in the orbital parameters as a function of the change in the planar orbital velocity. In $\S 3$ we determine the positional deviation of a perturbed orbit relative to an unperturbed orbit. Next, in $\S 4$ we calculate the uncertainty in the deflection distance resulting from the uncertainty in the orbital velocity change. In $\S 5$ we translate the positional uncertainties into confidence intervals that successful deflection is achieved. Finally, in $\S 6$ we incorporate this work into 
basic spacecraft intercept mission planning to determine confidence of deflection success.

\section{ORBITAL DYNAMICS}

\subsection{Unperturbed Orbit}

For completeness and to define quantities for later use, we begin by considering the case of an unperturbed planar orbit using the equations and notation from Ch. 2 of Murray \& Dermott (2000). The general expressions for the position $\vec{r}$ and planar velocity $\vec{v}$ of a body in orbit are

$$
\vec{r}=r \hat{r}, \quad \vec{v}=\dot{r} \hat{r}+r \dot{\theta} \hat{\theta}
$$

where $\hat{r}$ and $\hat{\theta}$ are the unit vectors along and perpendicular to the position vector $\vec{r}$. The orbit radius $r$, radial velocity $\dot{r}$ and angular velocity $r \dot{\theta}$ at any given point in the orbit can be expressed as

$$
\begin{aligned}
r & =\frac{a\left(1-e^{2}\right)}{1+e \cos (\theta-\omega)} \\
\dot{r} & =\sqrt{\frac{\mu}{a\left(1-e^{2}\right)}} e \sin (\theta-\omega) \\
r \dot{\theta} & =\sqrt{\frac{\mu}{a\left(1-e^{2}\right)}}(1+e \cos (\theta-\omega)) .
\end{aligned}
$$

Here $a$ is the semi-major axis distance, $e$ is the eccentricity, $\omega$ is the argument of pericenter, $\theta$ is the angle counterclockwise from the reference direction, and $\mu=G M_{\odot}$, with $G$ being the gravitational constant and $M_{\odot}$ the mass of the Sun. The true anomaly angle $f$, the angle from perihelion to the orbiting body's position as seen by the Sun, is

$$
f \equiv \theta-\omega .
$$

Note that one has the freedom to define $\omega=0$ initially such that $f=\theta$. A more useful quantity when examining the time dependence of an orbit is the eccentric anomaly, E (Murray \& Dermott 2000). The true anomaly is related to the eccentric anomaly $E$ by

$$
\tan \frac{f}{2}=\sqrt{\frac{1+e}{1-e}} \tan \frac{E}{2} .
$$

Rewriting the orbit radius given in Eq. 2 in terms of $E$ gives,

$$
r=a(1-e \cos E)
$$

where the $x$ and $y$ components of the position are

$$
\left(\begin{array}{l}
x \\
y
\end{array}\right)=\left(\begin{array}{c}
a(\cos E-e) \\
a \sqrt{1-e^{2}} \sin E
\end{array}\right) .
$$

The orbital position as a function of time is given by Kepler's Equation,

$$
E-e \sin E=\sqrt{\frac{\mu}{a^{3}}}(t-\tau)
$$

where $\tau$ is usually defined as $\tau=t$ at a pericenter passage, $E=0$, but can be shifted to any convenient value. Finally, the specific angular momentum $h$ and specific total energy $\varepsilon$ in terms of the orbital parameters are,

$$
\begin{aligned}
h & =\sqrt{\mu a\left(1-e^{2}\right)} \\
\varepsilon & =-\frac{\mu}{2 a} .
\end{aligned}
$$

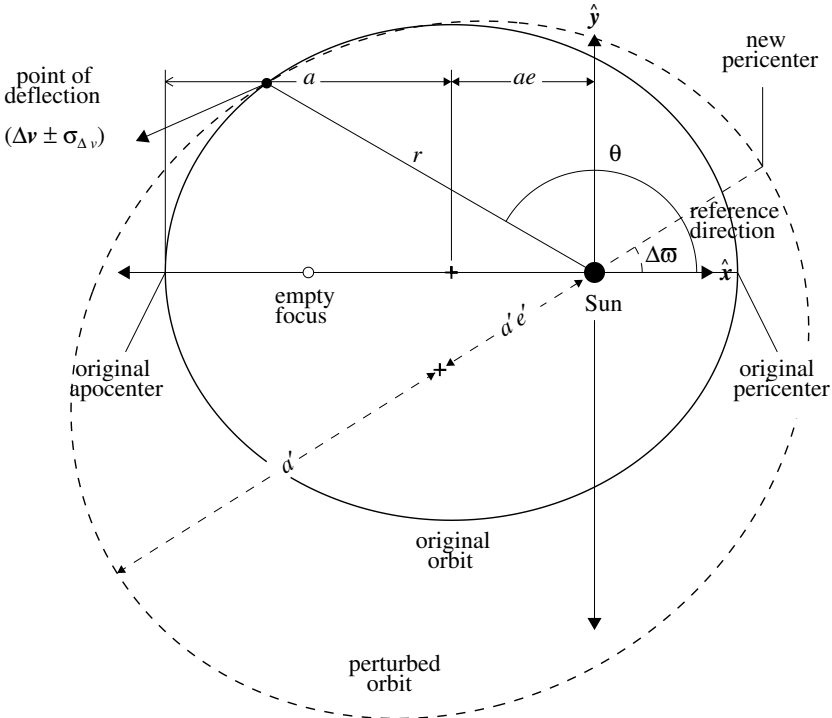

Figure 2. Diagram of the orbital components relevant for deflection analysis. The original orbit is the solid black line, with the impulsively modified orbit the dashed black line. The original orbital parameters $(a$ and $e)$ as well as the new orbit parameters $\left(a^{\prime}\right.$, $e^{\prime}$, and $\Delta \omega$ ) are shown along with the point of deflection. Note that the scenario depicted here involves a much larger $\Delta v$ than would actually be employed and has been exaggerated for emphasis.

\subsection{Perturbed Orbit}

If we apply a perturbation at a point $f_{0}$ resulting in a planar, instantaneous velocity change $\Delta \vec{v}$ to an object that has initial orbital parameters $a$ and $e$ (where we have defined our axes such that $\omega=0$ initially), then the object will be shifted into a new orbit given by semimajor axis $a^{\prime}$, eccentricity $e^{\prime}$ and argument of pericenter $\omega^{\prime}=\Delta \omega$. Such an event is shown in a greatly exaggerated sense in Fig. 2. This new orbit will be in the same plane as and, critically, will share a common spacetime point $r_{0}$ with the original orbit: that of the deflection event at $f_{0}$.

A planar change in velocity $\Delta \vec{v}$ resulting from an instantaneous perturbation can be expressed in terms of the radial and angular components of the velocity change $\left(\Delta v_{r}, \Delta v_{\theta}\right)$, or, equivalently, in the direction of motion of and perpendicular to components $\left(\Delta v_{\|}, \Delta v_{\perp}\right)$,

$$
\begin{aligned}
\Delta \vec{v} & =\Delta v_{r} \hat{r}+\Delta v_{\theta} \hat{\theta} \\
& =\Delta v_{\|} \hat{v}_{\|}+\Delta v_{\perp} \hat{v}_{\perp}
\end{aligned}
$$

where the two are related by,

$$
\begin{aligned}
\Delta v_{r} & =\frac{\Delta v_{\|} e \sin f_{0}-\Delta v_{\perp}\left(1+e \cos f_{0}\right)}{\sqrt{1+2 e \cos f_{0}+e^{2}}} \\
\Delta v_{\theta} & =\frac{\Delta v_{\|}\left(1+e \cos f_{0}\right)+\Delta v_{\perp} e \sin f_{0}}{\sqrt{1+2 e \cos f_{0}+e^{2}}}
\end{aligned}
$$


or inversely,

$$
\begin{aligned}
\Delta v_{\|} & =\frac{\Delta v_{r} e \sin f_{0}+\Delta v_{\theta}\left(1+e \cos f_{0}\right)}{\sqrt{1+2 e \cos f_{0}+e^{2}}} \\
\Delta v_{\perp} & =\frac{\Delta v_{r}\left(1+e \cos f_{0}\right)-\Delta v_{\theta} e \sin f_{0}}{\sqrt{1+2 e \cos f_{0}+e^{2}}} .
\end{aligned}
$$

Using the standard equations for angular momentum and energy, the net change in the specific angular momentum and specific energy resulting from the velocity change is simply:

$$
\begin{aligned}
& \Delta h=h^{\prime}-h_{0}=|\vec{r} \times(\vec{v}+\Delta \vec{v})-\vec{r} \times \vec{v}| \\
& \Delta \varepsilon=\varepsilon^{\prime}-\varepsilon_{0}=\frac{1}{2}(\vec{v}+\Delta \vec{v})^{2}-\frac{1}{2} v^{2} .
\end{aligned}
$$

where $\vec{r}$ and $\vec{v}$ are the orbit radius and velocity vectors, respectively. Rewriting Eqs. 17 and 18 in terms of the perturbation parameters yields,

$$
\begin{aligned}
& \Delta h=r_{0} \Delta v_{\theta} \\
& \Delta \varepsilon=\frac{1}{2} \Delta v^{2}+v_{r_{0}} \Delta v_{r}+v_{\theta_{0}} \Delta v_{\theta}
\end{aligned}
$$

where $r_{0}, v_{r_{0}}$, and $v_{\theta_{0}}$ are the orbit radius, radial velocity and angular velocity, respectively, at the point of deflection $f_{0}$ (see Eqs. 2 - 4).

As shown in Eqs. 22 and 25 in Burns (1976), the time rate of change in the orbital elements $a$ and $e$ due to time rate changes in energy $\varepsilon$ and angular momentum $h$ are determined by merely differentiating Eqs. 10 and 11 with respect to time,

$$
\begin{aligned}
\frac{\mu \dot{a}}{2 a^{2}} & =\dot{\varepsilon} \\
\frac{e \dot{e}}{e^{2}-1} & =\frac{\dot{h}}{h}+\frac{\dot{\varepsilon}}{2 \varepsilon} .
\end{aligned}
$$

Since we assume that the energy and angular momentum of the system are changed instantaneously, we can integrate Eqs. 21 and 22 to obtain the nonlinear relationship between $\left(a^{\prime}, e^{\prime}\right)$ and $(a, e, \Delta \vec{v})$,

$$
\begin{aligned}
a^{\prime} & =a+\Delta a=-\frac{\mu}{2(\Delta \varepsilon+\varepsilon)} \\
e^{\prime} & =e+\Delta e \\
& =\left[1-\frac{\left(1-e^{2}\right)(h+\Delta h)^{2}(\varepsilon+\Delta \varepsilon)}{h^{2} \varepsilon}\right]^{1 / 2}
\end{aligned}
$$

An alternative and equivalent derivation of these quantities can be performed purely algebraically using Eqs. 10 and 11 and defining differences for $a$ and $e$ in those equations using $\Delta h$ and $\Delta \varepsilon$.

The new argument of pericenter, $\omega^{\prime}$ can be determined using Eq. 2. Using the definitions $\omega=0$ for the unperturbed orbit and that the perturbed orbit is required to pass through the point of deflection $\left(r_{0}, f_{0}\right), \omega^{\prime}$ can be solved for algebraically,

$$
\omega^{\prime}=\Delta \omega=f_{0}-\cos ^{-1}\left[\frac{1}{e^{\prime}}\left(\frac{a^{\prime}\left(1-e^{\prime 2}\right)}{r_{0}}-1\right)\right] .
$$

Note that while the solutions given in Eqs. 23 and 24 for $a^{\prime}$ and $e^{\prime}$ are valid for perturbations applied in any direction, the solution in Eq. 25 for $\omega^{\prime}$ is only valid when the perturbation is applied in the plane of motion.

The time from perihelion to the point of deflection for the original orbit can be determined using Eq. 9, where the choice of zero time is arbitrary. Thus, we define $t=0$ at the deflection point $E_{0}$ and solve for the time since perihelion at the deflection time,

$$
\tau_{0}=-\sqrt{\frac{a^{3}}{\mu}}\left(E_{0}-e \sin E_{0}\right) .
$$

For the new, perturbed orbit, the time from its perihelion $\omega^{\prime}$ to the point of deflection $f_{0}$, can be determined by solving for the new eccentric anomaly $E_{0}^{\prime}$ using Eq. 7,

$$
\begin{aligned}
E_{0}^{\prime} & =\cos ^{-1}\left[\frac{1}{e^{\prime}}\left(1-\frac{r_{0}}{a^{\prime}}\right)\right] \\
\tau_{0}^{\prime} & =-\sqrt{\frac{a^{\prime 3}}{\mu}}\left(E_{0}^{\prime}-e^{\prime} \sin E_{0}^{\prime}\right) .
\end{aligned}
$$

These equations for a perturbed orbit are derived in the technical limit of a delta function impulsive force, and are very good approximations for the impulsive deflections from nuclear devices and kinetic impactors (up to and including colliding planetesimals absent significant breakup effects). It should also be noted that certain other effects that occur very quickly compared to an orbital period can provide forces on the object that can be considered impulsive. Most notably in this category are the "keyhole" events due to 3-body effects (Valsecchi et al. 2003). During such an interaction a properly calibrated impulsive force can create the correct initial and final orbital parameters of the object for before and after the keyhole passage. Thus the presence of such occurrences can be accounted for in the above framework using a simple extension of the formalism to include multiple successive impulsive deflections, be they due to keyholes or man-made forces.

\section{POSITIONAL DEVIATIONS AT EARTH CROSSING TIMES}

Occasionally, an NEA will have an orbit that intersects or comes dangerously close to Earth's orbit. The NEA may be in or inclined relative to the plane of Earth's orbit. The NEA's initial, unperturbed orbit will cross Earth's orbit when $r=a_{\oplus}=1 \mathrm{AU}$, where we have approximated the orbit of Earth as circular. Using Eq. 7, the eccentric anomaly values for the NEA when it intersects Earth's orbit are,

$$
E_{\oplus}=\cos ^{-1}\left(\frac{a-a_{\oplus}}{a e}\right) .
$$

By approximating Earth's orbit as circular and defining $a_{\oplus} \equiv 1 \mathrm{AU}$, we introduce a relative systematic positional uncertainty of approximately $1.7 \%$, well below the scale of uncertainties from the selection of sources mentioned in $\S 1$. The corresponding $\left(x_{\oplus}, y_{\oplus}\right)$ positions are,

$$
\left(\begin{array}{l}
x_{\oplus} \\
y_{\oplus}
\end{array}\right)=\left(\begin{array}{c}
a\left(\cos E_{\oplus}-e\right) \\
a \sqrt{1-e^{2}} \sin E_{\oplus}
\end{array}\right) .
$$

If we anticipate an NEA impact at a given $E_{\oplus}$ and deflect it at an earlier point $E_{0}(t \equiv 0)$ in its orbit, then 
the time between the deflection and impact is,

$$
t_{\oplus}=\sqrt{\frac{a^{3}}{\mu}}\left(E_{\oplus}-E_{0}-e \sin E_{\oplus}+e \sin E_{0}\right),
$$

also know as the "epoch of interception" (as coined by Carusi et al. 2002). Rewriting Eq. 9 in terms of the epoch of interception, the shifted eccentric anomaly values $E_{\oplus}^{\prime}$ for a deflected NEA can then be solved for numerically,

$$
E_{\oplus}^{\prime}-e^{\prime} \sin E_{\oplus}^{\prime}=\sqrt{\frac{\mu}{a^{\prime 3}}}\left(t_{\oplus}-\tau_{0}^{\prime}\right) .
$$

The corresponding new $\left(x_{\oplus}^{\prime}, y_{\oplus}^{\prime}\right)$ positions at the epoch of interception are

$$
\left(\begin{array}{l}
x_{\oplus}^{\prime} \\
y_{\oplus}^{\prime}
\end{array}\right)=\left(\begin{array}{cc}
\cos \omega^{\prime} & -\sin \omega^{\prime} \\
\sin \omega^{\prime} & \cos \omega^{\prime}
\end{array}\right)\left(\begin{array}{c}
a^{\prime}\left(\cos E_{\oplus}^{\prime}-e^{\prime}\right) \\
a^{\prime} \sqrt{1-e^{\prime 2}} \sin E_{\oplus}^{\prime}
\end{array}\right)
$$

and the deflection distance is

$$
\Delta r=\sqrt{\left(x_{\oplus}-x_{\oplus}^{\prime}\right)^{2}+\left(y_{\oplus}-y_{\oplus}^{\prime}\right)^{2}}
$$

This result represents the fully nonlinear propagation of an arbitrary deflection delta-velocity $\Delta \vec{v}$ in the plane of the orbit of the NEA for the restricted two-body problem, and is an extension to the linearized theory given in Burns (1976). While we have chosen to use the epoch of interception as our figure of merit, the MOID can also be determined by finding the minimum distance between the Earth and the NEA as a function of time.

To evaluate the effect a chosen epoch of interception has on deflection distance in more detail, we choose to examine a fictitious object with the orbital parameters of 2011 AG5 ( $a=1.43$ and $e=0.39)$ that has been shifted slightly to impact the Earth on Feb. 4, 2040 (currently its date of closest approach). We call this object 2011 AG ${ }^{* 2}$. The effect the perturbation direction and point of deflection $E_{0}$ (and by extension epoch of interception $t_{\oplus}$, see Eq. 31) has on the deflection distance is shown in Fig. 3. Plotted are the distance per unit deflection delta-velocity as a function of the epoch of interception for perturbations in both the parallel and perpendicular directions of motion. At a given epoch of interception, the quantity $\Delta r / \Delta v$ is roughly linear for small changes in $\Delta v$. The degree to which the linear approximation is correct is discussed in great depth in Appendix A (with a companion discussion of the related question of the monotonicity of the equations, which is important for the uncertainty discussion below). For the time frame under consideration in this paper (chosen to be ten years for a realistic acquisition and deflection of a small- to medium-sized NEO) and for deflections on the tens of $\mathrm{cm} / \mathrm{s}$ level or below, the effect of nonlinearities will be $0.1 \%$ or less in the final deflection distance as defined here. For more information the reader is strongly urged to look at Appendix A.

One notes immediately that the deflection is more effective at certain points than others. These peaks correspond to deflection when the object is at perihelion, which results in a maximization of the change in energy

\footnotetext{
${ }^{2}$ We choose the fictitious object 2011 AG5* as an illustrative example, however, a detailed deflection plan for the real 2011 AG5 can be found in Yeomans et al. (2012).
}

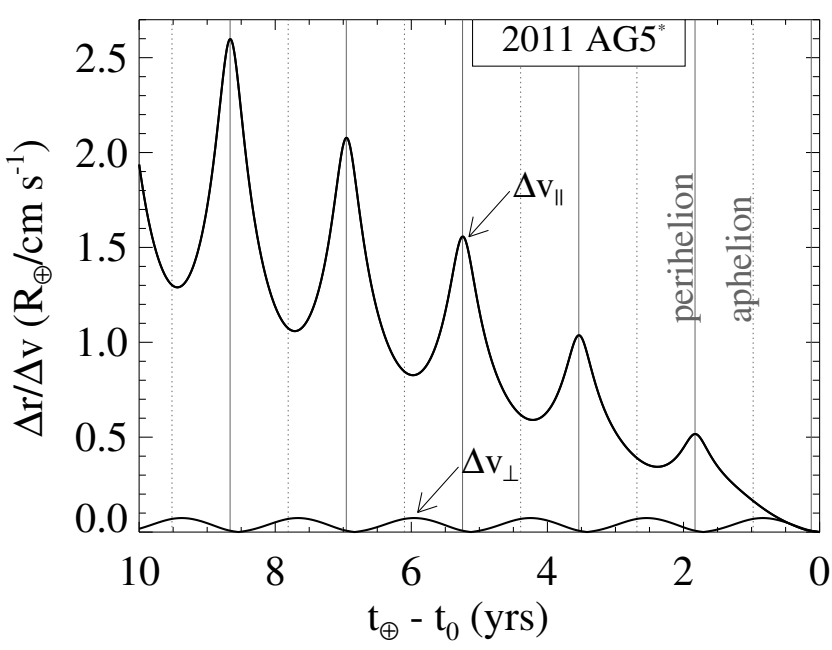

Figure 3. Example of positional deviations per unit deflection delta-velocity at the first Earth crossing for asteroid 2011 AG5* ( $a=1.431, e=0.3903)$ as a function of deflection delta-velocity magnitude, direction and time for small values of $\Delta v$. The $x$-axis shows epoch of interception, with the Earth crossing time $\left(t_{\oplus}\right)$ held fixed and the choice of deflection time $\left(t_{0}\right)$ varied. The $y$-axis gives the ratio of the resulting deflection distance (Earth radii, $R_{\oplus}$ ) to the deflection delta-velocity $\left(\mathrm{cm} \mathrm{s}^{-1}\right)$. The results for two orthogonal velocity components (perpendicular to and in the direction of motion) are shown. The dotted and solid vertical lines show the times of aphelion and perihelion crossings, respectively, for the unperturbed orbit. Perturbations in the direction of motion provide the largest positional changes at epoch of interception due to timing effects.

$\Delta U$ for a fixed $\Delta v$, a result that has been known for some time (e.g. Ahrens \& Harris 1992).

Fig. 4 shows a contour plot of the deflection distance per unit deflection delta-velocity $\Delta r / \Delta v$ for a range of NEA $a$ and $e$ orbital parameters and several discrete epochs of interception (1, 5 and 10 years). The left column shows the resulting deflection distance per unit deflection delta-velocity for perturbations in the parallel direction of motion, while the right column shows perturbations in the perpendicular direction of motion.

The cyclic nature of these plots is a function of the period of the orbit to the epoch of interception chosen. As orbits become more Aten-like (i.e. as $a$ decreases) the probability contours become increasingly narrow. One consequence of this is that small uncertainties in the semi-major axis distance can lead to large variations in the deflection efficiency. In the case of perturbations in the direction of motion (left column), deflections for a fixed small semi-major axis distance and higher eccentricities result in rapidly oscillating deflection efficiency, which is primarily due to the rapidly changing proximity of the orbital perihelion to the deflection attempt.

\section{UNCERTAINTY QUANTIFICATION}

In any realistic deflection attempt there will be multiple sources of possible uncertainty. The complete composition of the object may be poorly understood, including not only elemental composition, but porosity and heterogeneity of the interior as well. Indeed, the total mass of the object may not even be known to a high degree of accuracy if only the brightness of the object has been observed. Furthermore, the coupling of the deflection attempt with the object will add uncertainty to the final 

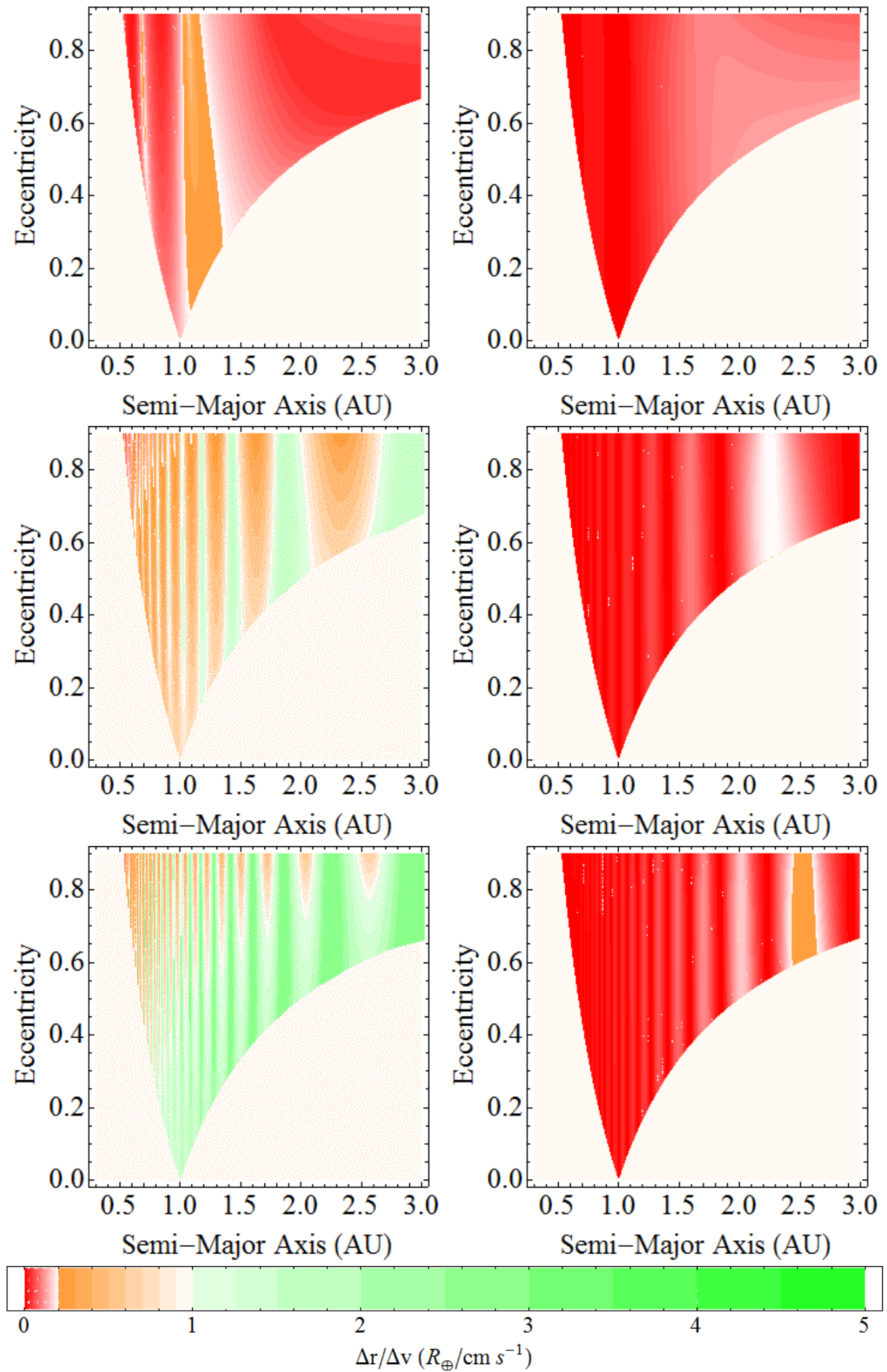

Figure 4. Deflection distance per unit deflection delta-velocity for a continuous range of NEA orbital parameters and discrete epochs of interception (from top to bottom: 1, 5 and 10 years). The left column shows the resulting deflection distance per unit deflection deltavelocity for perturbations in the parallel direction of motion, while the right column shows results for perturbations in the perpendicular direction of motion. 
velocity of the deflection. These unknowns will result in a final uncertainty in the deflection delta-velocity delivered to the object, and this uncertainty will propagate through the resulting orbit to create a final positional uncertainty associated with the deflection given in Eq. 34 .

Here, we consider uncertainties associated with the magnitude and direction of the impulse applied to the orbiting body. We begin by assuming a Gaussian uncertainty in the deflection delta-velocity $\Delta v$. It should be noted that while we have assumed a Gaussian distribution for simplicity of exposition, this is not strictly necessary and in fact any distribution in $\Delta v$ can be propagated though the above equations. For a Gaussian uncertainty in the deflection delta-velocity $\sigma_{\Delta v}$, the $1 \sigma$ (i.e. $68 \%$ confidence interval) uncertainty level in the deflection distance $\sigma_{\Delta r}$ can be computed in the normal way:

$$
\sigma_{\Delta r}^{2} \approx \sum_{i} \sigma_{\Delta v_{i}}^{2}\left(\frac{\partial \Delta r}{\partial \Delta v_{i}}\right)^{2}
$$

where $i \in\{r, \theta, \|, \perp\}$.

The derivative of the deflection distance at Earth-orbit crossing with respect to the deflection delta-velocity used in Eq. 35 can be determined by taking the derivative of Eq. 34 with respect to $\Delta v$,

$$
\frac{\partial \Delta r}{\partial \Delta v_{i}}=\frac{x_{\oplus}^{\prime}-x_{\oplus}}{\Delta r} \frac{\partial x_{\oplus}^{\prime}}{\partial \Delta v_{i}}+\frac{y_{\oplus}^{\prime}-y_{\oplus}}{\Delta r} \frac{\partial y_{\oplus}^{\prime}}{\partial \Delta v_{i}},
$$

where the derivatives of $x_{\oplus}^{\prime}$ and $y_{\oplus}^{\prime}$ components with respect to $\Delta v_{i}$ are determined by taking the derivative of Eq. 33 with respect to $\Delta v$,

$$
\begin{aligned}
\left(\begin{array}{c}
\frac{\partial x_{\oplus}^{\prime}}{\partial \Delta v_{i}} \\
\frac{\partial y_{\oplus}^{\prime}}{\partial \Delta v_{i}}
\end{array}\right) & =\left(\begin{array}{c}
-\sin \omega^{\prime}-\cos \omega^{\prime} \\
\cos \omega^{\prime}-\sin \omega^{\prime}
\end{array}\right)\left(\begin{array}{c}
a^{\prime}\left(\cos E_{\oplus}^{\prime}-e^{\prime}\right) \\
a^{\prime} \sqrt{1-e^{\prime 2}} \sin E_{\oplus}^{\prime}
\end{array}\right) \frac{\partial \omega}{\partial \Delta v_{i}} \\
& +\left(\begin{array}{c}
\cos \omega^{\prime}-\sin \omega^{\prime} \\
\sin \omega^{\prime} \cos \omega^{\prime}
\end{array}\right)\left[\left(\begin{array}{c}
\cos E_{\oplus}^{\prime}-e^{\prime} \\
\sqrt{1-e^{\prime 2}} \sin E_{\oplus}^{\prime}
\end{array}\right) \frac{\partial a^{\prime}}{\partial \Delta v_{i}}\right. \\
& -\left(\begin{array}{c}
a^{\prime} e^{\prime} \sin E_{\oplus}^{\prime} \\
\sqrt{1-e^{\prime 2}}
\end{array}\right) \frac{\partial e^{\prime}}{\partial \Delta v_{i}} \\
& \left.+\left(\begin{array}{c}
-a^{\prime} \sin E_{\oplus}^{\prime} \\
a^{\prime} \sqrt{1-e^{\prime 2}} \cos E_{\oplus}^{\prime}
\end{array}\right) \frac{\partial E_{\oplus}^{\prime}}{\partial \Delta v_{i}}\right]
\end{aligned}
$$

If a perturbation $\Delta \vec{v}$ is applied at some point $f_{0}$ in an orbit, then the derivative of the change in angular momentum and energy with respect to the change in the radial and angular orbital velocities are,

$$
\begin{array}{ll}
\frac{\partial \Delta h}{\partial \Delta v_{r}}=0 & \frac{\partial \Delta h}{\partial \Delta v_{\theta}}=r_{0} \\
\frac{\partial \Delta \varepsilon}{\partial \Delta v_{r}}=v_{r}+\Delta v_{r} & \frac{\partial \Delta \varepsilon}{\partial \Delta v_{\theta}}=v_{\theta}+\Delta v_{\theta}
\end{array}
$$

where $v_{r}$ and $v_{\theta}$ are the radial and angular orbital velocities at the point of deflection given in Eqs. 3 and 4, respectively. The derivatives of the individual orbital pa- rameters with respect to $\Delta v_{i}$ are

$$
\begin{aligned}
& \frac{\partial a^{\prime}}{\partial \Delta v_{i}}=\frac{\mu}{2 \varepsilon^{\prime 2}} \frac{\partial \Delta \varepsilon}{\partial \Delta v_{i}} \\
& \frac{\partial e^{\prime}}{\partial \Delta v_{i}}=\frac{h^{\prime}}{\mu^{2} e^{\prime}}\left(h^{\prime} \frac{\partial \Delta \varepsilon}{\partial \Delta v_{i}}+2 \varepsilon^{\prime} \frac{\partial \Delta h}{\partial \Delta v_{i}}\right) \\
& \frac{\partial \omega^{\prime}}{\partial \Delta v_{i}}=\frac{1}{r_{0} e^{\prime} \sin \left(f_{0}-\omega^{\prime}\right)} \times \\
& {\left[\left(1-e^{2}\right) \frac{\partial a^{\prime}}{\partial \Delta v_{i}}+\frac{r_{0}-a^{\prime}\left(1+e^{\prime 2}\right)}{e^{\prime}} \frac{\partial e^{\prime}}{\partial \Delta v_{i}}\right]} \\
& \frac{\partial E_{\oplus}^{\prime}}{\partial \Delta v_{i}}=\frac{1}{1-e^{\prime} \cos E_{\oplus}^{\prime}} \times \\
& {\left[\left(-\frac{3}{2} \sqrt{\frac{\mu}{a^{\prime 5}}} t_{\oplus}-\frac{r_{0}^{2}}{a^{\prime 3} e^{\prime} \sin E_{0}^{\prime}}\right) \frac{\partial a^{\prime}}{\partial \Delta v_{i}}\right.} \\
& \left.+\left(\sin E_{\oplus}^{\prime}-\sin E_{0}^{\prime}+\frac{r_{0}}{a^{\prime} e^{\prime} \tan E_{0}^{\prime}}\right) \frac{\partial e^{\prime}}{\partial \Delta v_{i}}\right]
\end{aligned}
$$

By evaluating Eqs. 36 through 45 in combination one can arrive at the fully propagated Gaussian uncertainty for the deflection distance in Eq. 35. One notes that the uncertainties in either the $x$ or $y$ components could be computed in a similar fashion using just the results from Eq. 37. This allows the user to determine the full 2D uncertainty envelope for the perturbed orbit at the epoch of interception. However, it should be noted that for deflections in the direction of motion (nominally the more effective choice) this envelope will be highly skewed in the direction of motion at the time of intercept, with very little uncertainty perpendicular to the direction of motion. One can show this from the equations given, though this is left as a simple geometric exercise to the reader.

While the uncertainty propagation inherent in Eq. 35 takes the standard form, it should be noted that this formula is only an approximation to the true uncertainty. This approximation is based on the assumption of nearlinearity of $\Delta r$ with respect to $\Delta v$ in the neighborhood of $\left\{\Delta v-\sigma_{\Delta v}, \Delta v+\sigma_{\Delta v}\right\}$. The degree to which the equations under consideration are nonlinear is discussed at length in Appendix A.

This nonlinearity is related to questions concerning the monotonicity of Eq. 34, which are also examined in Appendix A. In the case of Eq. 35 it is shown that the function is monotonic in $\Delta v$ over an extremely wide range of possible input values, including values far beyond the scope of any deflection mission. With this knowledge in hand one can evaluate the fully nonlinear uncertainty propagation simply by evaluating the function in Eq. 35 at the boundaries of the confidence level one is interested in. More specifically, for a $68 \%$ confidence interval in $\Delta v$ represented by $\Delta v \pm \sigma_{\Delta v}$ the corresponding fully nonlinear $68 \%$ uncertainty interval for $\Delta r(\Delta v)$ will be given by $\left\{\Delta r\left(\Delta v-\sigma_{\Delta v}\right), \Delta r\left(\Delta v+\sigma_{\Delta v}\right)\right\}$. In the limit of a completely linear function in $\Delta v$ this interval will be the same as $\Delta r \pm \sigma_{\Delta r}$. Given the large degree to which our function is linear in $\Delta v$ both methods will give effectively equivalent results for the relatively small $\Delta v$ 's needed for a deflection mission, however for the figures in the remainder of this paper the fully nonlinear propagation is used. 
Using the fictitious object 2011 AG5* outlined in $\S 3$, we examine the $1 \sigma$, uncertainty envelope associated with a $2 \mathrm{~cm} / \mathrm{s}$ mean deflection velocity with a $10 \%$ uncertainty for deflections in the direction of and perpendicular to the direction of motion for epochs of interception out to ten years. An uncertainty in the deflection delta-velocity of $10 \%$ is chosen to be illustrative and does not necessarily represent the "true" uncertainty. These results are shown in Fig. 5. As noted previously, the deflection is most effective at perihelion and in the direction of motion, and the uncertainty envelope in those time periods is larger as well. In contrast, the overall deflection for a perpendicular $\Delta v$ is so small that the associated uncertainty envelope cannot be distinguished from the line itself.

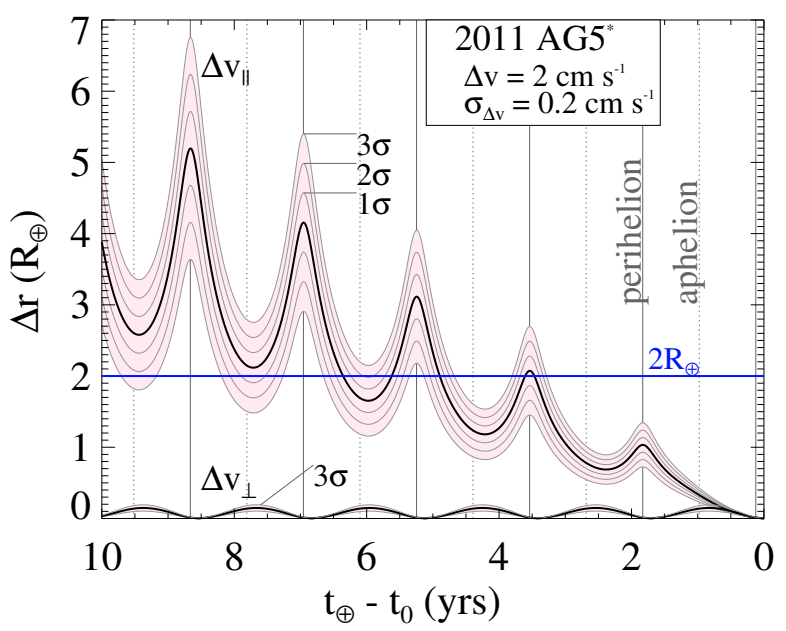

Figure 5. The deflection distance (in Earth radii, $R_{\oplus}$ ) for our fictitious object 2011 AG5* as a function of epoch of interception, with associated 1,2 , and $3 \sigma$ confidence intervals (shaded regions). This curve corresponds to a $2 \mathrm{~cm} / \mathrm{s}$ mean deflection with a $10 \%$ uncertainty (chosen to be illustrative), for deflections in both the parallel- and perpendicular-to-direction-of-motion directions (top and bottom curves, respectively). The blue horizontal line is a $2 R_{\oplus}$ deflection level, which nominally corresponds to a successful deflection attempt (NRC 2010).

\section{CONFIDENCE LEVELS}

To simplify the discussion from here on we consider velocity changes with or against the direction of motion of the object, not perpendicular to, which are the preferred mode of impulsive deflection at epochs of interception greater than a few months, as is evident from Fig. 3 (Vasile \& Colombo 2011). As stated previously, we also assume the uncertainty in the deflection delta-velocity $\Delta v$ to be Gaussian distributed.

For a given deflection scenario, the probability of a successful deflection can be characterized by a confidence function. To illustrate, we consider a deflection scenario in the direction of motion using our fictitious object 2011 AG5* where a $2 \mathrm{~cm} / \mathrm{s}$ deflection with a $10 \%$ uncertainty is applied. Fig. 5 shows the resulting deflection distance (black line) and the 1,2 , and $3 \sigma$ confidences intervals (shaded regions) as a function of epoch of interception. Specifying a minimum acceptable deflection distance, roughly $2 R_{\oplus}$ (horizontal blue line, NRC 2010),

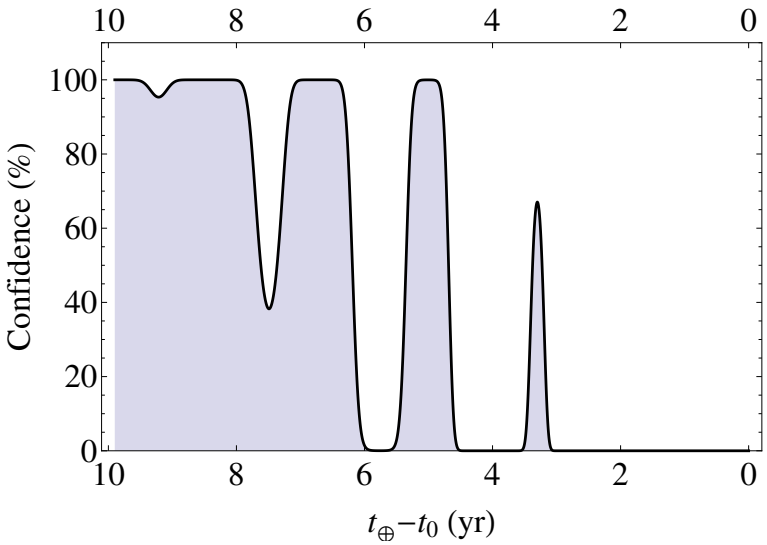

Figure 6. The percent probability that an object with the orbital parameters of 2011 AG5, when given a $2 \mathrm{~cm} / \mathrm{s}$ impulse with $10 \%$ uncertainty, will be deflected by $2 R_{\oplus}$ or greater, as a function of epoch of interception (see the horizontal $2 R_{\oplus}$ line in Fig. 5).

one can quantitatively examine the percent confidence one has in a specific deflection attempt. Specifically, one can take the distribution function for $\Delta r$ (the contours for which at 1, 2, and $3 \sigma$ are shown in Fig. 5) and integrate over it for values above $2 R_{\oplus}$, resulting in a total probability that the deflection attempt for that object at that epoch of interception will be greater than $2 R_{\oplus}$. As a visual example, for times where the blue line crosses the bottom grey $3 \sigma$ line in Fig. 5 (for example near 5.25 years out) the probability will be $99 \%$, corresponding to the $3 \sigma$ confidence level.

Performing the calculation discussed above results in the confidence function shown in Fig. 6. Note that significant confidence is achieved at greater epochs of interception. At a time of deflection nearing 10 years there is a comfortably high chance of mission success, while prior to 3 years there is a depressing probability of failure. At an epoch of interception slightly greater than 3 years there is a "last chance" window with a success probability of $\sim 70 \%$. Deflection attempts made after this window are likely to fail, unless a push harder than $2 \mathrm{~cm} / \mathrm{s}$ is applied. The earlier the deflection attempt the greater chance of success it generally has (as the change in velocity has a greater time over which to grow the change in the asteroid position), but with the propagation of the uncertainties through the orbital motion one can be more quantitative about this statement.

For an object with different orbital parameters the confidence function will change, which is illustrated in Fig. 7. For a deflection attempt at an epoch of interception of 10 years, the confidence levels for a $2 R_{\oplus}$ deflection or greater are shown, with darker blue regions showing higher confidence levels and white regions showing a confidence level near zero. Immediately, it can be seen that asteroids whose perihelion just reaches Earth's orbit and asteroids with Earth-like orbits have consistently high probabilities of successful deflection, while Aten asteroids (asteroids whose aphelion just reaches Earth's orbit) have success probabilities that are highly dependent on the initial orbital parameters.

Holding the eccentricity $e$ fixed and varying the semimajor axis distance $a$, one can see that the probability of success exhibits an oscillatory pattern. This structure is primarily due to the differences in orbital period (which 


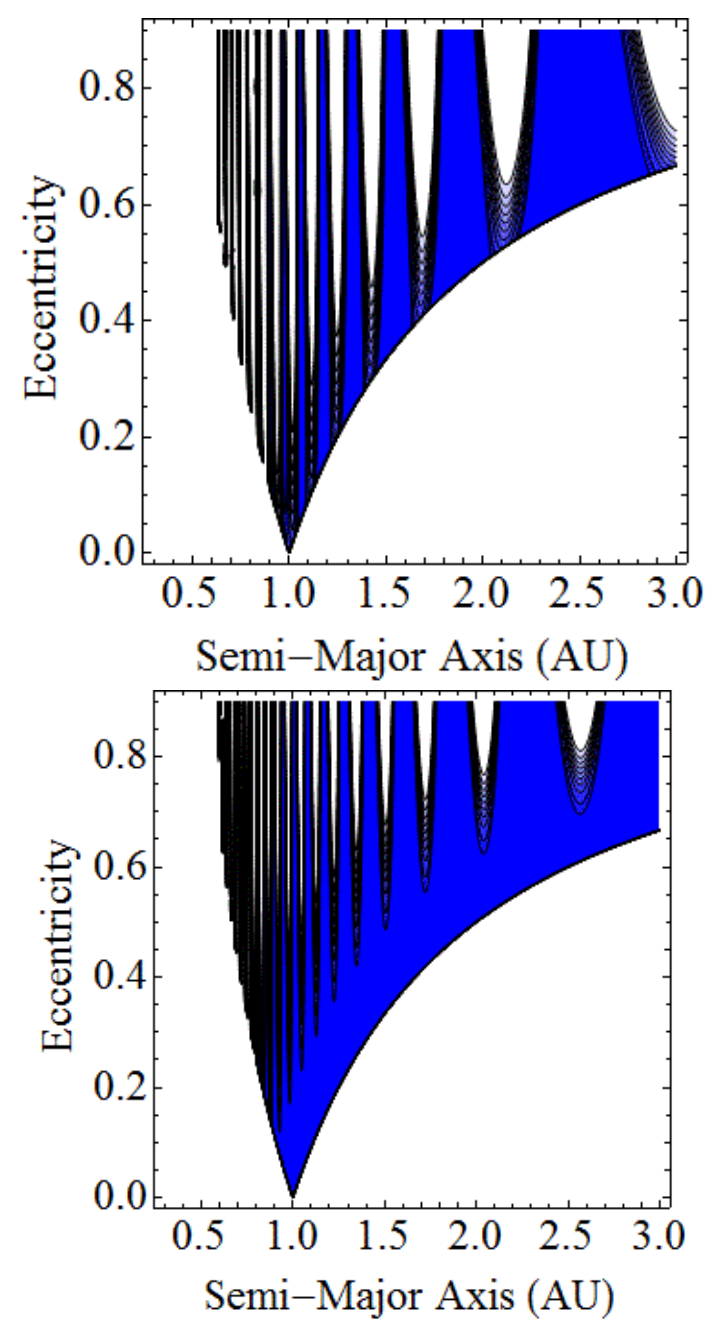

Figure 7. Contour plots of the confidence level for a mean deflection of $2 \mathrm{~cm} / \mathrm{s}$ and $10 \%$ uncertainty, epochs of interception 7.5 (top) and 10 (bottom) years, for changing eccentricity and semimajor axis. Darker blue regions are higher probability. The shape with respect to the semi-major axis is primarily due to the changes to the orbital period as the semi-major axis changes, and the proximity (in time) of the deflection attempt to that object's perihelion passage.

is a function of only $a$ ), as the locations of the vertical finger-like structures shift along the horizontal axis as the epoch of interception is varied. The exact placement of these structures is determined by the proximity of that orbit with respect to its perihelion passage (proximity in time at the epoch of interception chosen that is). Note that at small values of $a$, the orbits with opportunities for a successful deflection are in windows that become increasingly narrow. As $a$ is increased, these windows are widened but become fewer in number.

Changes in the eccentricity for a fixed semi-major axis distance are monotonic in nature. As eccentricity is increased, the confidence of a successful deflection decreases, though the rate at which the confidence decreases depends highly on the value of $a$. This, coupled with the 'finger-like' structure observed in the contours with changing a raises some interesting conclusions regarding the uncertainties due to the orbital determination of the NEA. Uncertainties in the eccentricity of the object will generally make only a small difference in the

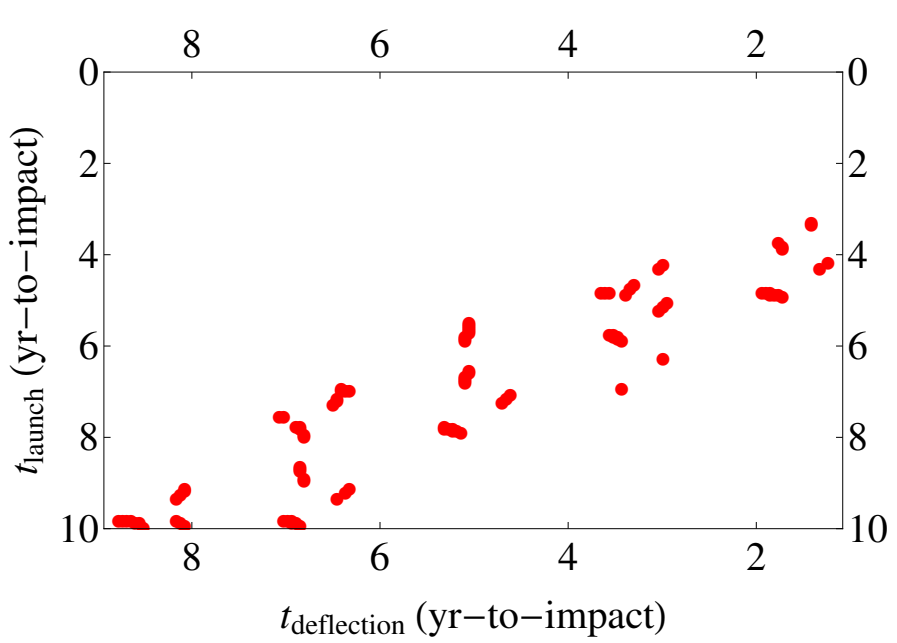

Figure 8. Launch time (in years to impact) vs. arrival time (i.e. the epoch of interception) for a flyby (nuclear) deflection mission to our fictitious object 2011 AG5*. This assumes an impact date of Feb. 4, 2040.

timing of the deflection opportunity. However, uncertainties in the semi-major axis distance will contribute a significantly larger uncertainty, especially at small $a$ as for the Aten family of asteroids.

Thus it is of vital importance that the semi-major axis of the object be known correctly prior to a deflection attempt, as significant changes in timing may be necessary. However, included in this discussion of the uncertainties needs to be folded information about the probability of an impact given uncertainties in the orbital elements of the object. The same orbital dynamics that give rise to uncertainties in the deflection distance also reduce the calculated probability of impact. Indeed, the level of certainty of impact at which decision-makers may wish to make a deflection attempt may be such that the impact on the confidence level of the deflection is negligible. However, a full discussion of the interplay here between the technical nature of orbital uncertainties and policy decisions is beyond the scope of this report.

\section{MISSION PLANNING}

These confidence limits can be particularly valuable when used in conjunction with spacecraft mission planning, which any successful NEA deflection attempt must include. An important component of this involves launch dates and transit times for spacecraft missions to intercept a NEA. Potential launch and flyby dates for intercept missions can be found at NASA Trajectory Browser.

To illustrate the use of the confidence intervals in a mission planning sense, we will look at an attempted nuclear deflection mission on our fictitious object 2011 AG5*. For simplicity, we assume that the impulse is applied in the direction of motion. Using the NASA Trajectory Browser tool one can obtain launch and flyby (i.e. epoch of interception) dates for the object in the years leading up to the close approach date of 2011 AG5 of Feb. 4, 2040. One hundred flyby dates are obtained (using the largest upper bound allowed on the rocket burn required for the orbital insertion and striving for minimum travel time) and the launch and flyby dates are plotted in Fig. 8, where the timescales are in years until the impact date. The flyby dates cluster into several group- 
ings, which correspond to the closest approaches of 2011 AG5* with Earth The relationship between launch and arrival date has an overall roughly linear relationship, but within each grouping the exact relationship becomes highly nonlinear.

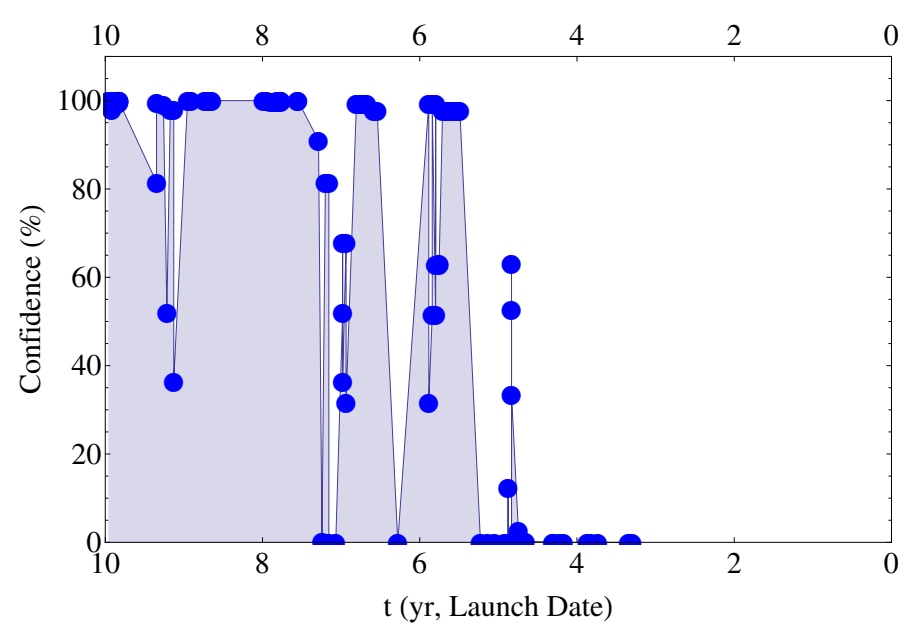

Figure 9. The percent probability that an object with the orbital parameters of 2011 AG5, when given a $2 \mathrm{~cm} / \mathrm{s}$ impulse with $10 \%$ uncertainty, will be deflected by $2 R_{\oplus}$ or greater, as a function of launch date (using the NASA Trajectory Browser). This assumes an impact date of Feb. 4, 2040. Note that launch dates varying by as little as a week can cause the confidence level to change by factors of two to three, due to differing flyby dates. Also note that the 'last chance' window launch dates all occur on the same day: April 7, 2035.

Mapping these flyby dates to their corresponding launch dates and using the confidence function in Fig. 6, one obtains the launch opportunities and corresponding probability of success shown in Fig. 9. One can see that launch dates varying by as little as a week can affect the confidence level by factors of two or three. Equally notably there are many launch dates that result in deflection attempts that will be almost completely unable to succeed. These results exemplify the highly nonlinear nature of the underlying orbital problem and propagation of uncertainty through the orbital motion, as well as the nontrivial coupling of launch dynamics with deflection dynamics. Most notably perhaps is the exceedingly narrow launch window for attempts that occur during the 'last chance' peak. All of these launches must occur on the same day: April 7, 2035 and are launched with differing insertion velocities.

In a real mission planning scenario the approach angle of the spacecraft relative to the NEA will need to be taken into consideration as uncertainties in this angle will introduce uncertainties. For example, consider both impact and nuclear deflection scenarios. The spacecraft will most likely have a restricted angle of approach due to its orbit trajectory. In the case of a nuclear standoff explosion, there may be some control over when the device is detonated, but the range of possible approach angles will be restricted by the orbit of the spacecraft and its ability to maneuver. In the impact case, there is less freedom as the impulse must occur when the spacecraft strikes the NEA. In a real scenario, the deflection deltavelocity and uncertainty for a range of approach angles can be determined by combining parallel and orthogonal deflection cases, again using the methodology developed in this paper.

\section{SUMMARY AND DISCUSSION}

We have calculated the probability that an impulsively deflected NEA will miss Earth as a function of the initial orbit and choice of deflection parameters for an impulsive orbital change due to either a massive kinetic impactor or a nuclear standoff burst. This is quantified through the development of the equations necessary for a fully nonlinear propagation of an impulsive orbital change through a deflected orbit. These equations allow one to express confidence of a successful deflection in terms of the uncertainties associated with the initial orbital parameters (due to limited observations, keyhole events, etc.), and/or uncertainties in the magnitude and direction of the applied impulse (due to unknowns in the NEA composition, efficiency of the energy coupling to the NEA, etc.).

We find impulses applied along the direction of motion are optimal for most scenarios, since the resulting deflection distance on average grows with time (in agreement with previous analytic and N-body work, Carusi et al. 2002; Izzo 2005; Vasile \& Colombo 2011). Furthermore, while the formalism derived is completely nonlinear, an analysis of the degree of nonlinearity (with respect to $\Delta v$ ) for the global parameter space of possible orbits was accomplished and shown to be small. This allows one to examine the problem in terms of deflection distance per unit $\Delta v$. For perturbations in the direction of motion, the biggest change in energy occurs when the body is perturbed at perihelion (as can be derived from simple energy arguments), where the difference between the deflection efficiency at perihelion and at aphelion can be greater than a factor of two. Consequently, it is often advantageous to wait and deflect an NEA when it is at perihelion rather than at an earlier point in its orbit. However, when the epoch of interception corresponds to less than one orbit, it is always preferable to act as soon as possible, at timescales on the order of a few months a deflection partially or totally in the perpendicular direction may be necessary (though it should be noted that on those timescales a disruption mission may be more advantageous).

As a function of the initial orbital parameters, the deflection efficiency was examined. The structure of the results is largely determined by the timing of the deflection attempt relative to its orbital perihelion, with monotonic changes tending toward decreasing efficiency at higher original eccentricities and highly oscillatory changes with increasing semi-major axis. The periodicity of these oscillations increases toward smaller semi-major axis objects (the Aten-like asteroids), leading to the conclusion that a correct, precise, determination of the semi-major axis for a threatening object from that region is of critical importance. Since there is still a significant need to understand the uncertainty distribution in the deflection delta-velocity independent of the orbit uncertainties, asteroid missions which accurately characterizes the orbit prior to deflection are crucial for getting a handle on the true deflection delta-velocity uncertainties associated with an impact event.

Using the equations for the deflected orbit, the un- 
certainty in the deflection distance as a function of the epoch of interception was derived as a function of initial orbit parameters, deflection delta-velocity and uncertainty in the deflection velocity. This was accomplished via two methods: first through the standard uncertainty propagation procedure looking at the function derivative in the neighborhood of the deflection parameter space and second through an examination of the nonlinearities of the function and an argument concerning functional monotonicity which then allowed for a propagation of the uncertainty including the full nonlinearity in $\Delta v$. Using these results, deflection confidence curves (defined as the probability to deflect an object by $2 R_{\oplus}$ or more for a given deflection delta-velocity and uncertainty) were examined for a specific case, that of a fictitious Earthcrossing asteroid which we name 2011 AG5*. In this scenario, a deflection delta-velocity of $2 \mathrm{~cm} \mathrm{~s}^{-1}$ with a $10 \%$ uncertainty is applied in the direction of motion at various epochs of interception, the uncertainties are propagated through the deflected orbit and the confidence interval is determined. We find that for a two Earth-radii deflection or greater, chances of failure are high when a deflection attempt is made at epochs of interception less than three years. Also, as the original $\Delta v$ uncertainty is increased the overall success probability decreases while the possible response windows broaden slightly.
Looking at the confidence levels across a range of possible orbits we found, similar to the deflection efficiency, a periodic structure in the semi-major axis that is largely reflective of the orbital period of the object and the temporal proximity of its perihelion crossing at the time of the deflection event. The rapid change of this characteristic for Aten-like asteroids at low values of the semimajor axis again emphasizes the need for precise knowledge of the orbital parameters in these cases before a deflection attempt can be reliably made. This statement is also true even for other orbits, as evidenced by the rapidity of the change in the confidence level with respect to possibly unknown orbital parameters.

Folding these results into a spacecraft mission planning scenario for our case study of 2011 AG5*, we found that for a reasonably successful deflection ( $>50 \%$ confidence), a spacecraft must be launched at $\geq 5$ years before Earth-impact. Also, the complex nonlinear relationship between possible launch dates and arrival times make for significant changes in confidence level for launch attempts that occur as little as a week apart. Fundamentally, the rapidity of the change in the confidence level, either with respect to timing or with respect to possibly unknown orbital parameters, underscores the need for a complete accounting of uncertainty sources in deflection attempts.

\section{Appendix A. NONLINEARITIES IN \& MONOTONICITY OF THE DEFLECTION DISTANCE EQUATION}

As the formalism outlined in this paper makes no inherent assumption about the 'smallness' of or linearity with respect to the $\Delta v$ applied to obtain the deflection distance it is useful to examine the nonlinearity in more detail. This will allow for a better understanding of the limits of the formalism and create the opportunity for a more precise determination of the the propagation of the uncertainty through the equations. We will first examine the limits of the formalism by looking at the places where the equations become discontinuous, zero, or infinite. We will then move on to a discussion of the degree to which the deflection distance is nonlinear in $\Delta v$ at values similar to those that would be used in an actual deflection event. This will naturally lead to a discussion about the degree to which the function is monotonic, with implications for the correct propagation of uncertainties through from the original $\Delta v$ to the final $\Delta r$.

\section{A1. Zeros, Discontinuities and Infinities}

We start by finding the zeros, discontinuities and infinities in Eqs. 42, 43, 44, and 45. Zeros and infinities occur in Eq 42 when any of the following are true,

$$
\begin{aligned}
& \frac{\partial a^{\prime}}{\partial \Delta v_{i}}=0 \quad\left\{\begin{array}{c}
\text { if } \frac{\partial \Delta \varepsilon}{\partial \Delta v_{i}}=0, \text { which occurs when } \\
\Delta v_{i}=-v_{i}, \quad \text { where } i=(r, \theta)
\end{array}\right. \\
& \frac{\partial a^{\prime}}{\partial \Delta v_{i}} \rightarrow \pm \infty\left\{\begin{aligned}
\text { if } \quad & \rightarrow-\varepsilon, \text { which occurs as } \\
\Delta v_{i} & \rightarrow v_{i} \pm \sqrt{v_{i}^{2}-\Delta v_{j}^{2}-2 v_{j} \Delta v_{j}+\frac{\mu}{a}} \\
\Longrightarrow v^{2} & \rightarrow\left(v_{i} \pm \sqrt{v_{i}^{2}-\Delta v_{j}^{2}-2 v_{j} \Delta v_{j}+\frac{\mu}{a}}\right)^{2}+\Delta v_{j}^{2} \\
\text { where } \quad(i, j) & =(r, \theta) \vee(\theta, r)
\end{aligned}\right.
\end{aligned}
$$

For the most part, deflection velocities are much less than the typical velocities in Eq. A1 \& A2. However, as $f_{0} \rightarrow n \pi$, orbital velocities in the $r$ direction begin to approach velocities of order the desired deflection. At these points in the orbit, particular care must be taken to ensure that a monotonic portion of the deflection delta-velocity space is being examined. However, one can always examine the limiting case as $f_{0} \rightarrow n \pi$ which will equal the real value at $f_{0}=n \pi$ due to the fundamentally continuous nature of the governing equations of motion. 
Zeros occur in Eq 43 when any of the following are true,

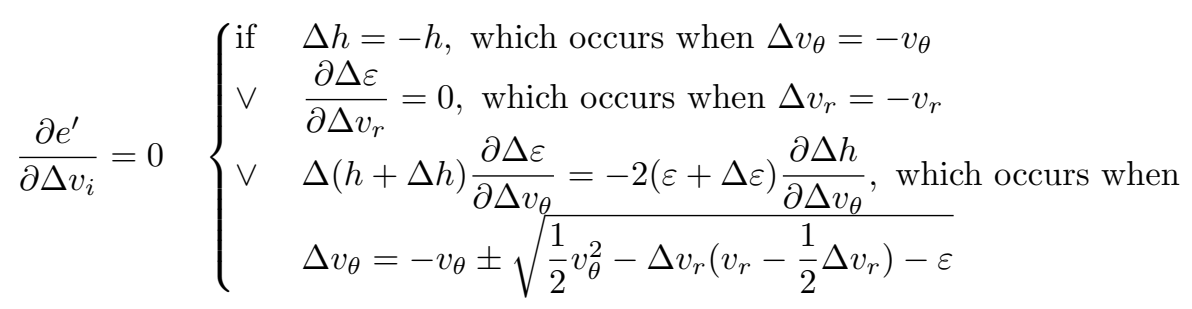

For the most part, deflection velocities are much less than the typical velocities in Eq. A3. However, as the point of deflection $f_{0}$ approaches angles of $n \pi$, inflection points in the $\left(\Delta v, e^{\prime}\right)$ curve approach velocities that are in the range of desired deflection velocities. These points correspond to circularizing the orbit. Eq. A3 is true when the point of deflection equals,

$$
f_{0}=2 \pi n+\cos ^{-1}\left[\sqrt{\frac{a\left(1-e^{2}\right)}{\mu e^{2}}}\left(-2 \Delta v_{\theta}+\sqrt{2\left(\Delta v_{\theta}^{2}-\varepsilon\right)-\Delta v_{r}\left(2 v_{r}+\Delta v_{r}\right)}\right)-\frac{1}{e}\right] .
$$

Discontinuities occur in Eq 44 when the following is true,

$$
\frac{\partial \omega^{\prime}}{\partial \Delta v_{i}}=0 \quad\left\{\text { if } \quad \frac{\partial e^{\prime}}{\partial \Delta v_{r}}=0 \text {, which occurs when } \Delta v_{\theta}=-v_{\theta} \vee \Delta v_{r}=-v_{r}\right.
$$

while discontinuities and infinities occur in Eq 45 for

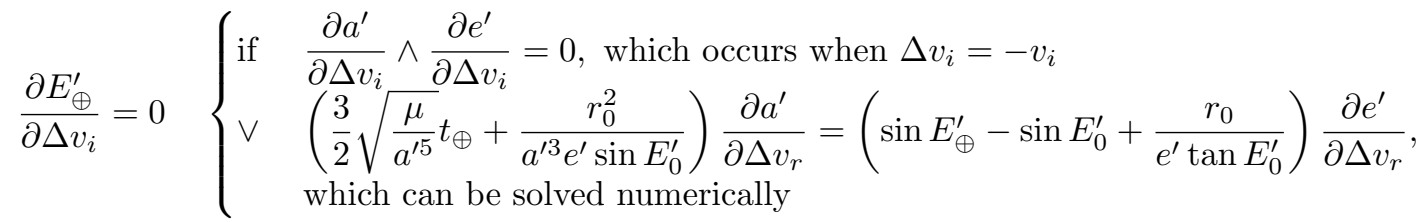

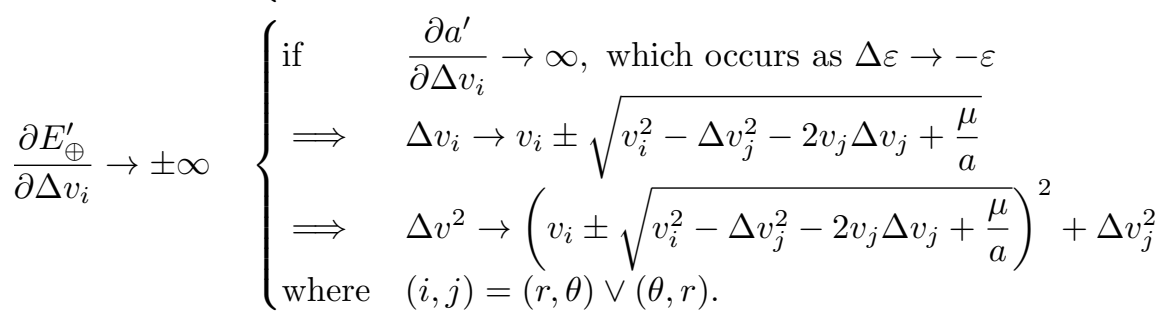

Eqs. A1, A3, A5 and A6 share zeros at $\Delta v_{i}=v_{i}$, meaning that $\partial \Delta r / \partial \Delta v=0$ is always true at $\Delta v_{i}=v_{i}$ for $i \in\{r, \theta\}$. This intuitively makes sense, as a deflection of that magnitude would fundamentally change the orbital character, usually resulting in a body free-falling into the sun. However, as orbital velocities are typically on the order of $\mathrm{km} / \mathrm{s}$ and most realistic deflection scenarios involve changes of around $\mathrm{cm} / \mathrm{s}$ we are well away from these limits of the formalism for most deflection scenarios.

\section{A2. Nonlinearities 83 Monotonicity in $\Delta v$}

For our application we know that this deflection will be a very small change, and to leading-order will be linear in $\Delta v$. By understanding how true this statement is, and more specifically by being able to compare the leading order (linear) term to the next-to-leading order (first nonlinear, $\Delta v^{2}$ ) term, one can both examine the degree of nonlinearity (i.e. the degree to which the simplifying linear approximation is correct) and determine the range of parameters over which the equations are monotonic (which will allow for a more complete understanding of the propagation of uncertainties in $\Delta v$ through to the $\Delta r$ equation).

We will examine this by looking at deflections in the parallel and perpendicular directions separately. It should be noted that the same approach could be taken for any arbitrary direction, but a separate analysis of the two most common limiting cases, that of a deflection parallel to the direction of motion and a deflection perpendicular to the direction of motion, will allow for a more straightforward analysis and span the space adequately. Taking the parallel direction first, one can write

$$
\begin{aligned}
& \Delta r_{\|}=a \cdot|\Delta v|+\frac{1}{2} b|\Delta v|^{2}+\mathcal{O}\left(|\Delta v|^{3}\right) \\
& a=\left.\left(\frac{\partial \Delta r_{\|}}{\partial|\Delta v|}\right)\right|_{|\Delta v|=0}, \quad b=\left.\left(\frac{\partial^{2} \Delta r_{\|}}{\partial|\Delta v|^{2}}\right)\right|_{|\Delta v|=0} .
\end{aligned}
$$




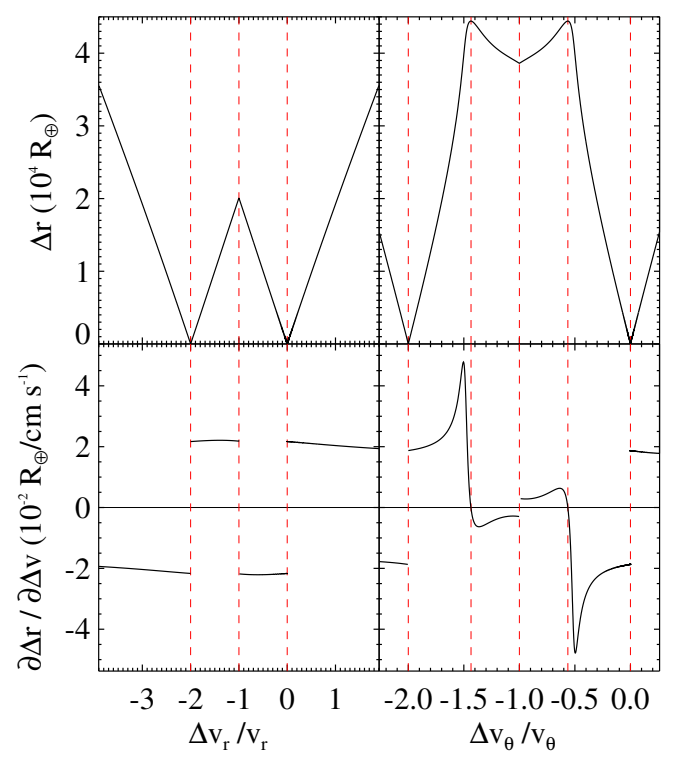

Figure A1. Discontinuities in the deflection distance equation and its derivative for a specific orbital case and deflection point (2011 AG5*: $\left.a=1.431 \mathrm{AU}, e=0.39, f_{0}=\pi / 3\right)$. The x-axis for each plot has been normalized by the appropriate orbital velocity component. From this it is easy to see that the discontinuities occur when the change in velocity zeros out or completely reverses a given component, which intuitively makes sense. As orbital velocities are typically on the order of $\mathrm{km} / \mathrm{s}$ or higher and most deflection scenarios involve changes on the order of $\mathrm{cm} / \mathrm{s}$ one can safely ignore the effect of these discontinuities for this case.

where this form is the standard expression of a Taylor expansion. One could similarly write

$$
\begin{aligned}
& \Delta r_{\perp}=c \cdot|\Delta v|+\frac{1}{2} d|\Delta v|^{2}+\mathcal{O}\left(|\Delta v|^{3}\right) \\
& c=\left.\left(\frac{\partial \Delta r_{\perp}}{\partial|\Delta v|}\right)\right|_{|\Delta v|=0}, \quad d=\left.\left(\frac{\partial^{2} \Delta r_{\perp}}{\partial|\Delta v|^{2}}\right)\right|_{|\Delta v|=0}
\end{aligned}
$$

where $a, b, c$, and $d$ will be complicated equations of the initial orbital parameters and the time of deflection. The values of these parameters as a function of the input parameters will give an indication of the degree of nonlinearity and, as discussed in more detail below, the degree to which the function can be taken to be monotonic.

While it would be possible for one to determine the analytic form of these coefficients, for the purposes of this exploration a less tedious numerical examination will be undertaken. Specifically, for the population of NEAs given in Fig. 1, a numerical calculation of the $\Delta r$ arising from a variety of $\Delta v$ values in both the parallel and perpendicular directions was calculated, for a set epoch of interception out to 10 years. For each orbit at each choice of epoch of interception the data as a function of $\Delta v$ was fit to a cubic in $\Delta v$. By fitting to a cubic one allows the higher order effects to be absorbed by the term of order $\Delta v^{3}$, thus giving convergence of the values for the coefficients of the lower order terms. For each orbit the epoch of interception yielding the most constraining value of the nonlinearity was then chosen, such that the resulting values of $a, b, c$, and $d$ that would be those for the 'most nonlinear' system, out to 10 years prior to impact.

The results of this methodology are shown in Fig. A2, with the deflection parallel to the direction of motion on the left and deflections perpendicular to the right. Note that the vertical axis in both figures is given as the logarithm of the ratio of the coefficients. This means, for example, that the value of $a / b$ at high eccentricities is $10^{4.5} \mathrm{~cm} / \mathrm{s} \approx 300 \mathrm{~m} / \mathrm{s}$. This means that for a $\Delta v \approx 1 \mathrm{~cm} / \mathrm{s}$ (a nominal deflection value) the ratio of the linear term to the leading nonlinear term in the expansion will be roughly $10^{4}$ at high eccentricities, while for lower eccentricities it will be around $10^{6}$. For deflections perpendicular to the direction of motion there is less readily noticeable structure in the $a-e$ space, but with a similar conclusion that the nonlinear terms contribute at a level of perhaps one part in $10^{5}$. However, this analysis was only done out to 10 years prior to impact, where for much longer time horizons the degree to which the nonlinear terms contribute increases. The reader should therefore be cautioned to redo this analysis if data for epochs of interception larger than 10 years is required.

One can extend this analysis into the related area of the monotonicity of the function for $\Delta r$. Given the above forms, one can determine the bounds over which monotonicity is maintained by looking at

$$
\begin{aligned}
& \frac{\partial \Delta r_{\|}}{\partial|\Delta v|}=a+b|\Delta v|=0 \\
& \frac{\partial \Delta r_{\perp}}{\partial|\Delta v|}=c+d|\Delta v|=0 .
\end{aligned}
$$



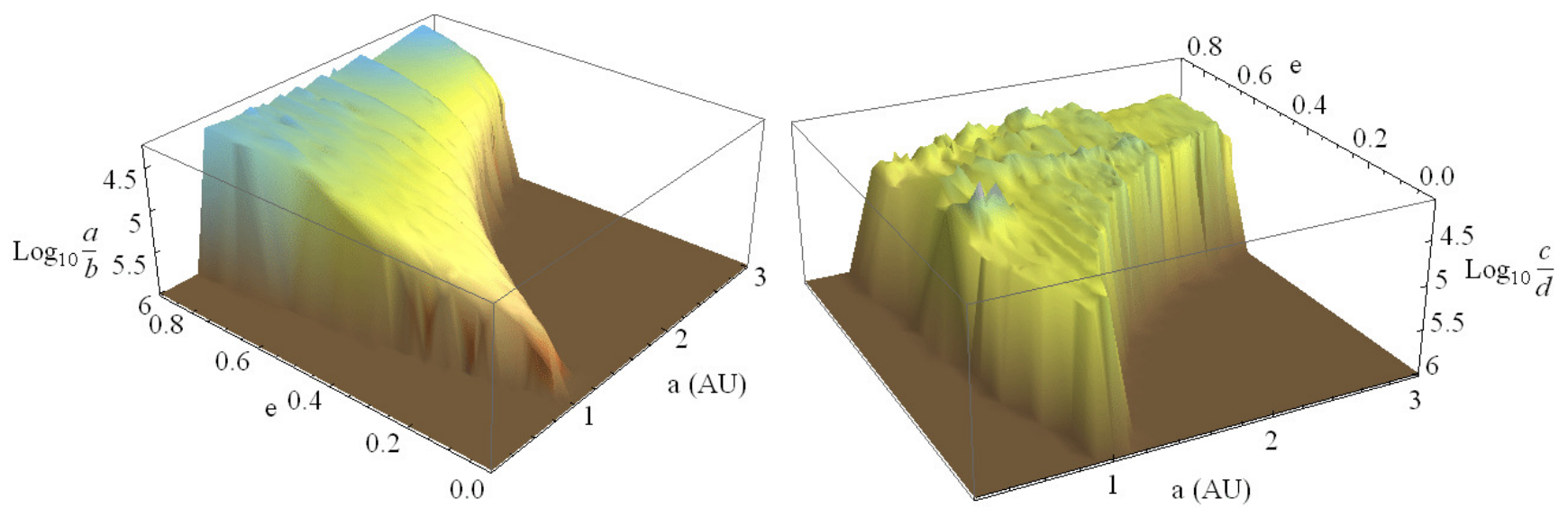

Figure A2. The negative of the $\Delta v$ value at which the function $\Delta r(\Delta v)$ is no longer monotonic. The results for deflections parallel to the direction of motion are shown at left, and perpendicular at right. These values of $a / b$ and $c / d$ reflect the most constraining values for a given orbit out to 10 years prior to impact. Note that even at high eccentricities this implies that the function is monotonic for all values of $\Delta v>-300 \mathrm{~m} / \mathrm{s}$, which is well away from the regime of nominal deflection velocities.

Solving these for $\Delta v$ one obtains the ratios $-a / b$ and $-c / d$, which are precisely what is plotted in Fig. A2. Thus, this will give the value for $\Delta v$ at which the deflection equations can no longer be safely assumed to be monotonic. More specifically, as long as the uncertainty quantification analysis being undertaken stays away from this boundary one case assume the equation is monotonic in $\Delta v$. Given that the most limiting case shown in Fig. A2 is at high eccentricities with a deflection parallel to the direction of motion, and that this boundary occurs at $-300 \mathrm{~m} / \mathrm{s}$ for the deflection delta-velocity, one can reasonably assume that for more nominal deflection velocities around $\pm 1 \mathrm{~cm} / \mathrm{s}$ that the equations are safely monotonic.

This analysis can be equally well applied to where the assumption of linearity in the deflection delta-velocity breaks down. Twice the value for $a / b$ given above is also the value where contributions from the term quadratic in $\Delta v$ become comparable in size to the contributions from the term linear in $\Delta v$. That is, for $\Delta v=2 a / b$ the magnitudes of the linear and quadratic terms in the expansion Eq. A8 are equal, and this occurs for a very wide range of orbits at a value of no less than $600 \mathrm{~m} / \mathrm{s}$ for the deflection. If one were to deflect an object with that velocity in the parallel-to-motion direction some very eccentric orbits would have comparable contributions from both terms. If a deflection one-tenth this size is applied then the term linear in $\Delta v$ will drop in magnitude by a factor of ten, while the quadratic term will drop by a factor of one hundred, making it a ten-percent variation in the linear approximation.

Given that most (human) deflection attempts will be in the $\mathrm{cm} / \mathrm{s}$ range, and that this represents a deflection deltavelocity smaller by a factor of more than three orders of magnitude than the above limits, one can then safely assume that the linear approximation will hold to within $0.1 \%$ for deflection attempts in the tens of $\mathrm{cm} / \mathrm{s}$ and below range. A similar analysis for deflections perpendicular to the direction of motion yields a slightly smaller perturbation. It should be noted that this is true only absent major N-body perturbations (such as keyhole events or other significant orbital variations) and only out to ten years in an object's orbit. However, in the realistic timeframe for the acquisition and deflection of a small- to medium-size NEO the majority of objects will not be affected by these limitations on the analysis.

One could ask, given that higher order terms are being neglected, how far can this analysis of monotonicity be believed? If a higher order term is added to the equation for $\Delta r_{\|}$(with a $\Delta v^{3}$ coefficient $b^{\prime} / 6$ ) and a similar derivation is run through, with an expansion in small powers of $b^{\prime}$ taken at the end one would obtain:

$$
\Delta v=-\frac{a}{b}\left(1-\frac{3 a b^{\prime}}{2 b^{2}}\right) .
$$

This extra term is itself of order $(\Delta v / v)^{2}$, meaning that the boundary given in Eq. A10 will be accurate to roughly within a factor of $(\Delta v / v)^{2}$, which is generally a small number.

LLNL-JRNL-643133. This work was performed under the auspices of the U.S. Department of Energy by Lawrence Livermore National Laboratory under Contract DE-AC52-07NA27344, and partially funded by the Laboratory Directed Research and Development Program at LLNL under tracking code 12-ERD-005.

The authors wish to recognize and acknowledge contributions from fellow LLNL Asteroid/Comet Mitigation group members: David Dearborn, James Elliott, Seran Gibbard, Rob Managan, Aaron Miles, Michael Owen, Eric Herbold, and the Principal Investigator, Paul Miller.

\section{REFERENCES}

Ahrens, T. J. \& Harris, A. W. 1992, Nature, 360, 429 Alvarez, L., Alvarez, W., Asaro, F. \& Michel, H. 1980, Science, 208, 1095 
Alvarez, W. \& Muller, R. A. 1984, Nature, 308, 718

Binzel, R. P., Xu, S., Bus, S. J. \& Bowell, E. 1992, Science, 257, 5071, 779

Boslough, M. B. E. \& Crawford, D. A. 2008, International Journal of Impact Engineering, 35, 1441

Bottke, W., et al. 2002, Icarus, 156, 399

Burns, J. A. 1976, American Journal of Physics, 44, 944

Carusi, A., Valsecchi, G. B., D’Abramo, G. \& Boattini, A. 2002, Icarus 159,417

Ćuk, M. \& Stewart, S. T. 2012, Science, 338, 1047

Edwards, W. N., Brown, P. G. \& ReVell, D. O. 2006, Journal of Atmospheric and Solar-Terrestrial Physics 68, 1136

Hildebrand, A., et al. 1991, Geology, 19, 867

Holdren, J., Office of Science and Technology Policy (OSTP) Response to Congress on near-Earth Objects, Letter, 15 Oct 2010

Holsapple, K. A. \& Housen, K. R. 2012, Icarus, 221, 2, 875

The International Astronomical Union (IAU) Minor Planet Center, PHA Close Approaches to the Earth, http://www.minorplanetcenter.net/iau/lists/PHACloseApp.html, 2014

Izzo, D. 2005, 15th AAS/AIAA Space Flight Mechanics Conference, AAS 05-141, Copper Mountain, Colorado, 2005

Johnson, K., Relative Orbit Elements for Satellites in Elliptical Orbits. Air Force Institute of Technology Thesis, 2010

Jet Propulsion Laboratory (JPL) http://neo.jpl.nasa.gov/news/fireball_130301.html, 1 March 2013

Jet Propulsion Laboratory (JPL), Small-Body Database Search Engine, http://ssd.jpl.nasa.gov/sbdb_query.cgi

Morbidelli, A., et al. 2000, Meteoritics and Planetary Science, 36, 6,1309

Morrison, D. 1992, The Spaceguard Survey: Report of the NASA International near-Earth-Object Detection Workshop, NASA, Washington, D.C.

Murray, C. D. \& Dermott, S. F. 2000, Solar System Dynamics (Cambridge, UK: Cambridge Univ. Press)
National Aeronautics and Space Administration (NASA) 2005, Authorization Act of 2005, Washington DC: National Aeronautics and Space Administration

National Aeronautics and Space Administration (NASA) 2007 near-Earth Object Survey and Deflection Analysis of Alternatives, Washington DC: National Aeronautics and Space Administration

National Aeronautics and Space Administration (NASA) 2008, Authorization Act of 2008, Washington DC: National Aeronautics and Space Administration

NASA, http://trajbrowser.arc.nasa.gov/index.php, 11 March 2013

National Research Council (NRC) 2010, Defending Planet Earth: near-Earth Object Surveys and Hazard Mitigation Strategies (Washington, DC: University of Arizona Press)

Owen, T. \& Bar-Nun, A. 1995, Icarus, 116, 215

Shafer, et al. 1994, "The Coupling of Energy to Asteroids and Comets." Hazards Due to Comets and Asteroids, Ed. Tom Gehrels, Mildred Shapley Matthews, and A. M. Schumann (Tucson: University of Arizona Press)

Sherrill, R. E., Sinclair, A. J., Sinha, S. C. \& Lovell, T. A. Time-varying transformations for Hill-Clohessy-Wiltshire solutions in elliptic orbits. Celest. Mech. Dyn. Astr. 119: 55-73, 2014

Shoemaker, E. M. 1983, Annual Review of Earth and Planetary Sciences, 11, 461, 461

Valsecchi, G. B. and Milani, A. and Gronchi, G. F. \& Chesley, S. R., 2004, A\&A 408, 1179-1196

Vasile, M. \& Colombo, C. 2011, eprint arXiv:1104.4670

Yeomans, D.K., Chodas, P.W., Sitarski, G., Szutowicz, S., Krolikowska, M., 2004. Cometary orbit determination and nongravitational forces. In: Festou, M.C., Keller, H.U., Weaver, H.A. (Eds.), Comets II, pp. 137-151.

Yeomans, D., et al. 2012, Report on Asteroid 2011 AG5 Hazard Assessment and Contingency Planning, NASA, Washington, D.C. 